\title{
Artículos cientificos, tipos de investigación y productividad cientifica en las Ciencias de la Salud
}

\author{
Scientific Articles, Types of Scientific Research and Productivity in Health Sciences \\ Artigos cientificos, tipos de investigação e produtividade cientifica nas ciências da saúde \\ Víctor Patricio Díaz-Narváez PhD' , Aracelis Calzadilla Núñez MD² \\ Recibido: 27 de marzo de $2015 \bullet$ Aceptado: 16 de agosto de 2015 \\ Doi: dx.doi.org/10.12804/revsalud14.01.2016.10
}

Para citar este artículo: Díaz-Narváez VP, Calzadilla-Núñez A. Artículos cientifícos, tipos de investigación y productividad cientifica en las ciencias de la salud. Rev Cienc Salud. 2016;14(1): 115-121. doi: dx.doi.org/10.12804/revsalud14.01.2016.10

\section{Resumen}

El objetivo del presente trabajo es describir diferentes tipos de Investigación Científica y su importancia dentro de la investigación formativa, la formación investigativa y la investigación propiamente dicha en las Ciencias de la Salud. Se plantea que algunos artículos son rechazados porque los autores no resuelven correctamente, desde el punto de vista metodológico, la concatenación que existe entre los tipos de investigación, los diseños de investigación, la clase de problemas inherentes a estos y los diseños de las investigaciones, los objetivos y los métodos que se deben proponer en estos contextos metodológicos.

Palabras clave: Metodología de la Investigación, investigación científica, tipos de investigación, Sociedad del Conocimiento, calidad de la investigación.

\section{Abstract}

The aim of this paper is to describe different types of useful research and its importance in the formative research, research training and research itself in health sciences. It is stated that one of the sources of these rejections could be because the authors did not resolve correctly, from a methodological point of view, the concatenation between the research types, research designs, the type of problems inherent to these types and research designs; objectives and methods should be proposed in these methodological contexts.

1 Facultad de Odontología, Universidad San Sebastián, Santiago Centro, Región Metropolitana, Chile Escuela de Kinesiología, Universidad Bernardo O'Higgins, Santiago, Chile.. Correo electrónico: victor.diaz@uss.cl

2 Universidad de Santiago de Chile. Departamento de Psiquiatría Infantil y del Adolescente, Hospital Félix Bulnes, Quinta Normal, Región Metropolitana, Chile. Correo electrónico: araceliscalza@gmail.com 
Keywords: Research methodology, Scientific research, Types of research, Knowledge society, Research quality.

Resumo

O objetivo do presente trabalho é descrever diferentes tipos de investigação científica e a sua importância dentro da investigação formativa, a formação investigativa e a investigação propriamente dita nas Ciências da Saúde. Expõe-se que alguns artigos são rejeitados porque os autores não resolvem corretamente, desde o ponto de vista metodológico, a concatenação que existe entre os tipos de investigação, os desenhos de investigação, o tipo de problemas inerentes a estes tipos e desenhos de investigações, os objetivos e os métodos que devem-se propor nestes contextos metodológicos.

Palavras-chave: Metodologia da Investigação, investigação científica, tipos de investigação, Sociedade do Conhecimento, qualidade da investigação.

\section{Introducción}

En Chile no existe pleno conocimiento de cuál es la función de la formación investigativa en la educación de los profesionales de la salud ni en cómo la actividad científica puede ser incorporada dentro de los procesos de enseñanzaaprendizaje en la educación superior en general $\mathrm{y}$, en particular, en las facultades de ciencias de la salud (medicina, odontología, enfermería, entre otras disciplinas) (1-3). Tal grado de conocimiento, a su vez, depende también del grado de aprehensión que tenga la universidad acerca de la Sociedad del Conocimiento (sc), de su papel, su rol y de las obligaciones de ella derivadas (4).

La SC exige de las universidades que, en la formación y en los procesos de enseñanzaaprendizaje implicados dentro de esta formación, garanticen la adquisición de un estilo de pensamiento independiente, crítico y con capacidad discriminativa y creadora. No es posible lograr este objetivo cuando, en la actualidad, no existe una política definida en torno a la incorporación de la estrategia investigativa como elemento importante en el currículum (pensum) de las carreras de la salud.

La falta de formación investigativa y la ausencia de una política clara, ya señaladas, pueden revelarse mediante los siguientes hechos: (a) la existencia de diferentes programas de metodología de la investigación científica en las distintas universidades, caracterizados por disímiles contenidos con heterogéneas profundidades o superficialidades conceptuales e, incluso, con la ausencia de este tipo de programas en el currículum de las carreras del área de la salud; (b) el proceso de enseñanza-aprendizaje de esta disciplina suele ser eminentemente teórico; (c) en los casos en que este programa se realice, la enseñanza y el aprendizaje de sus contenidos se caracterizan por la ausencia o una débil política longitudinal y sistémica en el currículum, capaz de consolidar esta actividad tanto teórica como práctica, a medida que el estudiante profundiza el conocimiento de la ciencia constituida, otorgada por los contenidos de las asignaturas en su carrera (1). 
Por otra parte, se conoce que existe una correlación positiva entre la calidad de los conocimientos, lograda por los estudiantes, durante el proceso de enseñanza-aprendizaje en la educación superior, y una política de participación constante y sistemática de los estudiantes en la praxis investigativa (2).

Este juicio parece ser concordante con lo señalado por otros autores (5-9) y que incluye también algunas de las resoluciones del Informe sobre la Conferencia Mundial de la Educación Médica de 1998 y ratificada en el año 2000 (8). Como consecuencia, la introducción del proceso investigativo en el currículum de las carreras de Medicina y otras disciplinas de la salud, a la sombra de las características de la Sc, no constituye una opción, como aparece declarado públicamente por algunas universidades, sino una necesidad insoslayable que deben asumir todas las universidades en forma responsable.

La correlación antes nombrada está, a su vez, relacionada, directamente, con el tipo de profesional que exige la sc $(1,3,9)$. Por tanto, la actividad investigativa debe ser un elemento vital para toda universidad que persiga adecuarse en esta esfera a dicha sociedad, y para preparar adecuadamente a los futuros profesionales médicos y de la salud. Sin embargo, la acción del profesional de la salud no se detiene con el ejercicio adecuado de su profesión, sino también con la capacidad de desplegar sus conocimientos y encontrar soluciones a problemas de salud en diferentes planos de complejidad y en diferentes contextos de su accionar profesional, toda vez que las Ciencias de la Salud, en su amplio espectro, acogen algunos campos del conocimiento estrechamente relacionados con las disciplinas sociales, como ocurre con la epidemiología y la psicología (tanto clínica como social) (10).

En otras palabras, las carencias de las competencias investigativas de pregrado contienen la potencialidad de afectar a la propia actividad profesional, la de posgrado, y en el estudio de especialidades médicas, odontológicas y de otras profesiones del área de la salud.

\section{Objetivo}

Describir diferentes tipos de investigación útiles y su importancia dentro de la investigación formativa, la formación investigativa y la investigación propiamente dicha en las Ciencias de la Salud.

\section{Desarrollo y discusión}

En general, no existe acuerdo en la clasificación de las distintas formas de investigación y, la que se asume por los diferentes autores, depende del paradigma epistemológico que sustentan. No obstante, algunos metodólogos parecen coincidir en que los tipos de investigación pueden ser clasificados como exploratorios, descriptivos, correlacionales y explicativos $(2,11-14)$.

Esta clasificación, cuando está concebida sobre la base de una premisa histórico-lógica, se asocia, directamente, con el estado del conocimiento que se tiene sobre un objeto de investigación en una disciplina científica concreta en un momento determinado $(2,10,12,14)$. Este trabajo asume tal premisa y constituye, por inferencia lógica, que es la principal clasificación o la clasificación de la que se derivan todas las demás formas de investigación $(2,10,14)$. Lo anterior, por una razón: el conocimiento científico siempre avanza (se mueve), en esencia, desde lo conocido hacia lo desconocido y, en torno a este movimiento, se derivan los demás tipos de investigación.

Como consecuencia, el primer tipo de investigación es la exploratoria, y se emplea esencialmente cuando el objeto de investigación se presenta ante los científicos como algo totalmente nuevo o insuficientemente conocido; por tanto, los estudios exploratorios tienen como 
función familiarizarse con objetos o fenómenos desconocidos o relativamente desconocidos y tratan de identificar, en estos, conceptos, características o variables promisorias (entre otros aspectos) que puedan potencialmente constituirse en futuras características específicas para estos objetos o fenómenos. Un ejemplo de investigación exploratoria puede encontrarse en los inicios de la epidemia del VIH (15).

La investigación descriptiva opera cuando se requiere delinear las características específicas descubiertas por las investigaciones exploratorias. Esta descripción podría realizarse usando métodos cualitativos y, en un estado superior de descripción, usando métodos cuantitativos. Estos últimos tienen como función esencial medir (de la forma más precisa posible) las características, propiedades, dimensiones o componentes descubiertos en las investigaciones exploratorias; de esta manera, los estudios exploratorios se interesan por descubrir, mientras que las investigaciones descriptivas, en última instancia, se interesan en medir con la mayor precisión posible.

Esto supone, que los científicos dominan completamente el potencial cognoscitivo de los métodos de medición empleados. Un ejemplo de investigación descriptiva constituyó la caracterización o descripción de la molécula de ADN. Este ejemplo demuestra la importancia de este tipo de investigaciones, cuando el conocimiento de los rasgos fundamentales sobre un objeto aún no está completo (no se refiere al ADN, sino a los procesos de la herencia genética). La descripción de esta molécula permitió, posteriormente, postular hipótesis interesantes sobre los procesos de la herencia y estimuló notoriamente el desarrollo de nuevas disciplinas científicas. Las investigaciones correlacionales intentan descubrir si dos o más conceptos o propiedades de objetos están aso- ciados, como es su forma de asociación y en qué grado o magnitud lo están.

En este tipo de investigaciones no siempre es posible identificar variables dependientes e independientes, y la información acerca de la posible causalidad se extrae de la ciencia constituida (teoría acumulada) con cierto grado de certeza o fundada sospecha acerca del factor causal. Por lo tanto, estas investigaciones tienen cierto valor explicativo, pero de carácter parcial. Sin embargo, siempre existirá el riesgo de encontrar dos variables relacionadas desde el punto de vista estadístico-matemático, pero de forma espuria.

Un ejemplo de este tipo de investigación es el que se da cuando se determinan factores de riesgo para ciertas patologías cardíacas: aparición de arritmias en la angina inestable aguda que tendría como factores de riesgo el antecedente personal de cardiopatía isquémica, seguido de hipercolesterolemia y de hipertensión arterial (16). De ahí, la importancia de contextualizar constantemente este tipo de investigaciones con la ciencia constituida (1). Por último, las investigaciones explicativas son aquellas que están destinadas al descubrimiento de las leyes esenciales que pueden dar cuenta del porqué existen tales o cuales propiedades y del porqué estas propiedades pueden asociarse entre sí.

En esencia, su interés se centra en explicar por qué ocurre un fenómeno, en qué condiciones se manifiesta o por qué dos o más variables están relacionadas. Estas investigaciones son más estructuradas que los demás tipos de estudios e implican los propósitos de todas ellas, lo que proporciona un profundo sentido de entendimiento del objeto o fenómeno que se está estudiando. Un ejemplo de este tipo de investigaciones son aquellos que tratan de conocer la causa última de la fuerza de gravedad. 
Como consecuencia, los tres primeros tipos de investigación se asocian fuertemente con los métodos empíricos del conocimiento científico, lo que significa que su función esencial es encontrar hechos científicos de diferentes órdenes de complejidad y, el último, se asocia fuertemente con los métodos teóricos del conocimiento, es decir, a los procesos mentales que permiten la construcción de juicios, hipótesis y leyes teóricas lógicas extraídas desde la ciencia constituida y de los hechos observados, pero con cierto grado de independencia de ella (17).

Como puede apreciar el lector, los métodos se subordinan a los tipos de investigación y no acontece el proceso inverso. Por otra parte, los tipos de investigación antes señalados se relacionan con tipos diferentes de problemas científicos. Si se acepta que un problema científico es la expresión del desconocimiento en la ciencia, entonces es posible relacionar que los distintos tipos de investigaciones tienen sus propias clases de problemas (objetivos y métodos), cuyos procesos de investigación específicos intentarán resolver problemas inherentes a ese tipo de investigación al emplear los métodos específicos para la solución de determinados problemas $(1,2,18)$.

Por esta razón, y ejemplificando, una investigación explicativa tiene problemas, objetivos y métodos propios que no tienen nada en común, a veces, con los otros tipos de investigación. La clasificación de las investigaciones en experimentales y no experimentales (diseños de investigación) se subordinan a los tipos de investigaciones descritas al inicio. Por tanto, a modo de ejemplo, una investigación exploratoria puede ser experimental o no experimental.

Se aprecia entonces que los tipos de investigación están lejos de ser una simple lista presentada en forma ordenada, pero, sin sentido sistémico, y desligados de los procesos de desarrollo natural del conocimiento científico.
La ausencia de la enseñanza de la concatenación jerárquica de los tipos de investigación es un factor de confusión en el aprendizaje de la Metodología de la Investigación Científica en Pregrado, Posgrado y Especialidades Médicas.

En más de una oportunidad, se ha observado que algunos colegas manifiestan desazón al recibir las respuestas de rechazo de sus artículos. Una de las fuentes de estos rechazos puede deberse a que los autores no resuelven correctamente, desde el punto de vista metodológico, la concatenación que existe entre los tipos de investigación, los diseños de investigación, el tipo de problemas inherentes a estos tipos y diseños de investigaciones, los objetivos y los métodos que se deben proponer en estos contextos metodológicos.

La ausencia del conocimiento de este ligamiento lógico, ya expuesto anteriormente, constituye una fuente de error en la correcta observación del instante de desarrollo del objeto cognoscente y de las herramientas metodológicas y métodos adecuados que se requieren para enfrentar con éxito la investigación (18).

En la tarea de eliminar todas estas fuentes de errores inciden varios factores que pueden coadyuvar a un buen desempeño investigativo. Por razones de espacio, es imposible enumerar o describir. Como consecuencia, de todo lo expuesto más arriba, la calidad de la investigación no puede medirse por el tipo de investigación que se realiza, sino por la correcta aprehensión del estado de desarrollo del conocimiento que se tiene sobre un objeto o fenómeno estudiado y por el empleo de todas las herramientas metodológicas existentes y adecuadas para ese estado de desarrollo ya nombrado.

De esta manera, una investigación descriptiva, por ejemplo, no será buena o mala en sí misma, sino que lo será porque el objeto ya ha sido descrito, o no, con anterioridad o porque 
se usaron, o no, las herramientas metodológicas correctas o porque se elaboraron, o no, los objetivos apropiados y coherentes con ese estado de desarrollo. Las investigaciones explicativas, por ejemplo, las cuales se asocian con investigaciones de punta (en todos los campos y disciplinas de la ciencia: filosofía, sociología, psicología, neuroanatomía, genética molecular, entre otras) no son buenas o malas por ser tales, sino porque existe suficiente información del objeto o fenómeno estudiado que permite intentar explicar la esencia de estos.

Lo anterior no es óbice para entender que las investigaciones explicativas son más potentes, desde el punto de vista cognoscitivo, que, por ejemplo, las exploratorias. Lo que se trata de explicar es que el tipo de investigación que sea escogido, en un momento dado, no depende del investigador ni de sus deseos o preferencias individuales, sino del estado real y concreto en que se encuentre el grado de conocimiento que se tiene sobre el objeto o fenómeno de interés para el investigador.

\section{Conclusiones}

El lector puede pensar que la exposición realizada conduciría a la concepción de que los tipos de investigación descritos son entes separados $\mathrm{y}$ sin vasos comunicantes ni intersecciones entre ellos. En realidad, la dialéctica de la ciencia es muy compleja. Así, por ejemplo, ninguna investigación es "puramente" exploratoria o descriptiva.

Se realiza la separación mecanicista (disección) de ellas con el objeto de comprender su individualidad, pero en la viva realidad del desarrollo del conocimiento humano, estos tipos de investigaciones (que no son más que una secuencia de acciones que determinan procesos específicos de investigación) pueden encontrar puntos de intersección. Por ejemplo, una investigación descriptiva puede implicar también la actividad exploratoria cuando en la intención de describir se puede descubrir una nueva característica del objeto o fenómeno estudiado. El "error" que acá se puede cometer, desde el punto de vista de la productividad científica, es mezclar ambas acciones en una misma investigación. El investigador debe ser capaz de separar estos dos momentos.

Por otra parte, es necesario enfatizar que es necesario respetar la forma de presentar los artículos, los cuales deben caracterizarse por la existencia de concisión, claridad y coherencia, aspectos que no surgen solo por una exigencia formal-estructural, diseñada por la "directrices para autores", sino por la estructura de pensamiento que exige la propia ciencia. Por último, los jueces de las revistas científicas pueden colaborar en esta tarea.

No es función de ellos transformarse en profesores, sin embargo, el hecho de explicar en forma exhaustiva, no solo los errores teórico-conceptuales y errores en la aplicación o decisión de métodos propios inherentes a la disciplina de la cual se trate el artículo, sino también los errores metodológicos y, si es posible, de los errores o insuficiencias estadísticas que podrían estar contenidas en el artículo rechazado, será de invaluable ayuda para los profesionales de la salud que intentan exponer sus hallazgos en revistas científicas. Una acción como esta podría ser un elemento más que contribuya al aumento de la cultura de la publicación $(19,20)$.

\section{Descargos de responsabilidad}

Ambos autores han participado en la generación de la concepción, confección, redacción, revisión crítica y aprobación final del manuscrito y asumen la responsabilidad de todas las ideas y concepciones que aparecen es este artículo. Declaran que ellos financiaron la investigación y que no tienen conflicto de interés. 


\section{Referencias}

1. Díaz VP. Relación entre la sociedad del conocimiento, Metodología de la Investigación Científica y producción científica estudiantil en estudiantes de medicina, Chile. Colomb Med. 2011;42(3):388-99.

2. Díaz V. Metodología de la investigación Científica y bioestadística para profesionales y estudiantes de Ciencias Médicas. Santiago, Chile: RiL Editores; 2009.

3. Peña F, Zapata MA. La docencia de la antropología física en la Escuela Nacional de Antropología e Historia frente a la sociedad del conocimiento y las políticas educativas. Est Antrop Biol. 2005;12:223-46.

4. Harrison L, Hernández AR, Cianelli R, Rivera MS, Urrutia M. Competencias en investigación para diferentes niveles de formación de enfermeras: Una perspectiva latinoamericana. Cienc Enferm. 2005;11:59-71.

5. Grilli M. La investigación y su relación con la educación médica. Rev Nefrol Dial Transpl. 2005;25:1-4.

6. D'Ottavio AE, Carrera LI. La ciencia y la investigación como herramientas en el proceso de enseñanza-aprendizaje. Rev Dig Univers. 2005;6(5):1-7.

7. Otero J, Barrios I, Prieto GA. El objetivo en el contexto de la dirección estratégica, el proceso docente y la investigación científica. Rev Cub. Salud Pública. 2006;32:(3).

8. Organización Mundial de la Salud. Resolución xII. Informe sobre la Conferencia Mundial de Educación Médica. Rev Cubana Educ Med Sup. 2000;14(2):206-9.

9. Clouet-Huerta DE, Correa K. Investigación Médica en Pregrado: ¿qué está sucediendo en Chile? Rev Med Chile. 2014;142:1488-90.

10. Díaz V, Calzadilla A. El reduccionismo, antirreduccionismo y el papel de los enfoques y métodos generales del conocimiento científico. Cinta Moebio. 2001;11:148-60.

11. Hernández R, Fernández C, Baptista MP. Metodología de la Investigación. 5. ed. México: McGraw Hill; 2014.

12. Jiménez R. Metodología de la Investigación. La Habana: Edit. Ciencias Médicas; 1998.

13. Behar DS. Metodología de la Investigación. Cabo Verde: Edit. Shalom; 2008.

14. Díaz-Narváez VP, Calzadilla A, López H. Una aproximación al concepto de hecho científico. Rev Austral de Cienc Soc. 2004;8:3-16.

15. Carrillo E, Villegas A. El descubrimiento del viH en los albores de la epidemia del sida. Rev Invest Clín. 2004;56(2):130-3.

16. Frómeta A, Sánchez S, Álvarez A, Fonseca JC, Quezada A, López C. Factores de riesgo en la aparición de arritmias en la angina inestable aguda. Rev Cub Med Int Emerg. 2009;8(2):1505-18.

17. Lewontin R. Las bases del conflicto en biología. En: Martínez S, Barahona A, (eds.). Historia y explicación en biología. México: Fondo de Cultura Económica; 1998. p. 96-106.

18. Díaz-Narváez VP. El concepto de ciencia como sistema, el positivismo, neopositivismo y las "investigaciones cuantitativas y cualitativas". Salud Uninorte 2014;30(2):227-44.

19. Díaz-Narváez VP. El "problema" de investigación científica: un problema no solucionado en pregrado. Rev Nefrol Dial Transpl. 2012;32(1):59-61.

20. Montenegro-Idrogo JJ, Huerta-Callado YM. Cultura de Publicación: realidades diferentes. Rev Med Chile. 2014;142(3):402. 